\title{
Automated Vessel Segmentation in CT and CTA scans of the Lung Via Deep Neural Networks
}

wenjun tan ( $\square$ tanwenjun@cse.neu.edu.cn )

Northeastern University https://orcid.org/0000-0002-3840-9528

luyu zhou

Northeastern University

xiaoshuo li

Northeastern University

xiaoyu yang

Tongji University

yufei chen

Tongji University

jinzhu yang

Northeastern University

\section{Research}

Keywords: segmentation, pulmonary, vessel, U-Net, network, CT images, CTA

Posted Date: May 25th, 2021

DOI: https://doi.org/10.21203/rs.3.rs-551102/v1

License: (c) (i) This work is licensed under a Creative Commons Attribution 4.0 International License.

Read Full License 


\section{Abstract}

Background: The distribution of pulmonary vessels in computed tomography images is important for diagnosing disease, formulating surgical plans and pulmonary research. However, there are many challenges of pulmonary vascular segmentation due to its characteristics of narrow and long pipes, discrete distribution and tree-like structure. With the development of deep learning and medical image processing, an automatic, accurate and fast segmentation algorithm of pulmonary blood vessels becomes possible.

Methods: Based on the International Symposium on Image Computing and Digital Medicine 2020 challenge pulmonary vascular segmentation task, this paper objectively evaluates the performance of 12 different algorithms in chest computed tomography and computed tomography angiography. First, we present the annotated reference dataset including computed tomography and computed tomography angiography. Second, by analyzing the advantages and disadvantages of each team's algorithm from 12 different institution, the reasons for some defects and improvements are summarized. Finally, we discuss the ways and methods to improve the results.

Results: These methods were compared with the ground truth by the numerical results and the intuitive results from computed tomography and computed tomography angiography images. Most methods do an admirable job in pulmonary vascular extraction, with dice coefficients ranging from 0.70 to 0.85 , and the dice coefficient for the top three methods are about 0.80 .

Conclusions: These results show that the methods which consider spatial information, fuse multi-scale feature map, or have an excellent post-processing are significant for further improving the accuracy of pulmonary vascular segmentation. Keywords: segmentation; pulmonary; vessel; U-Net; network; CT images; CTA

\section{Full Text}

This preprint is available for download as a PDF.

\section{Figures}

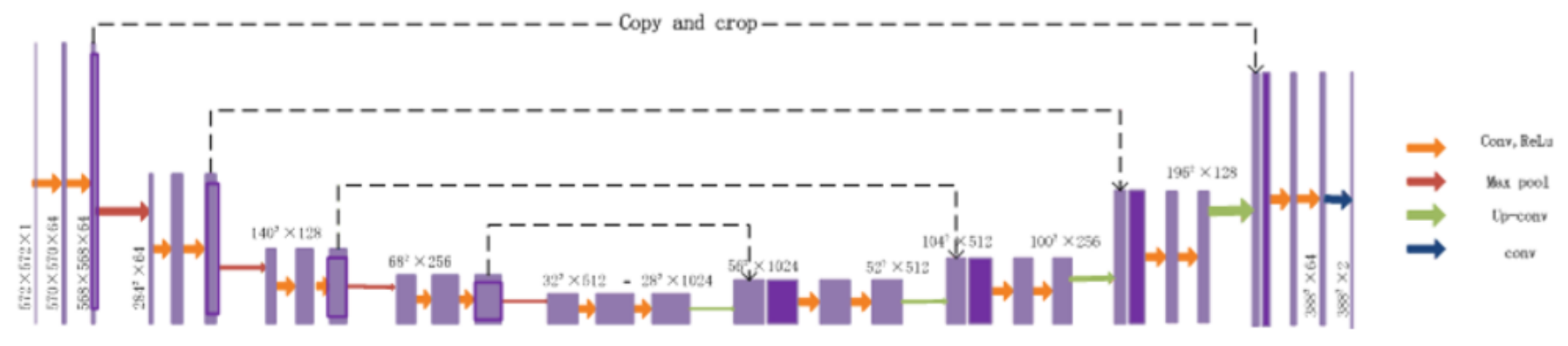

Figure 1 
U-net architecture (example for 32x32 pixels in the lowest resolution).

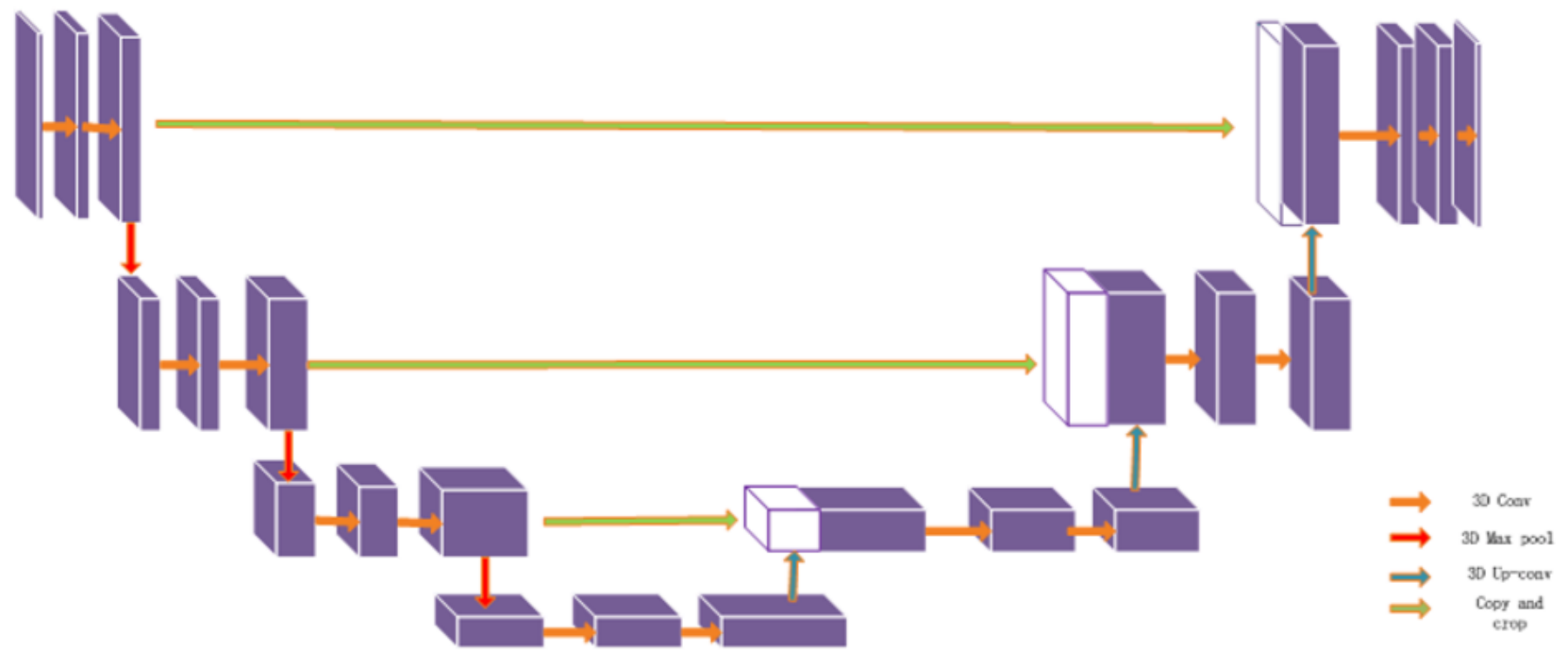

Figure 2

3D U-net architecture.

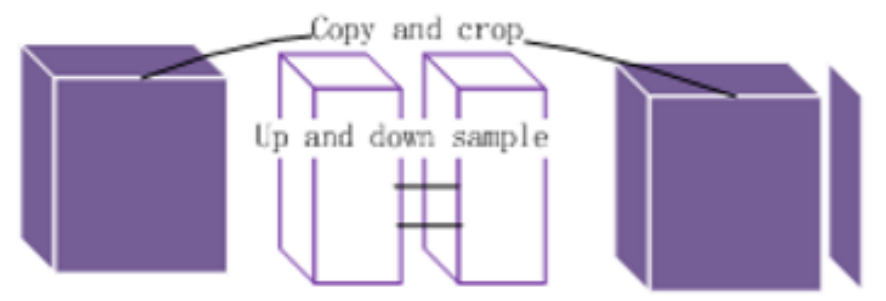

Stage 1
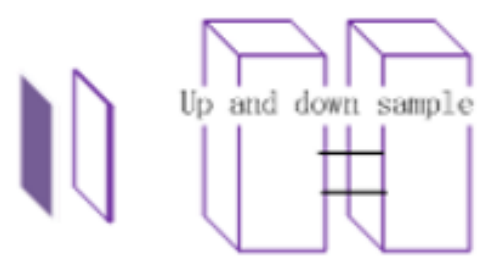

Stage 2

\section{Figure 3}

nnU-net architecture. Stage 1: a 3D U-Net processes downsampled data, the resulting segmentation maps are upsampled to the original resolution. Stage 2: these segmentations are concatenated as one-hot encodings to the full resolution data and refined by a second 3D U-Net. 

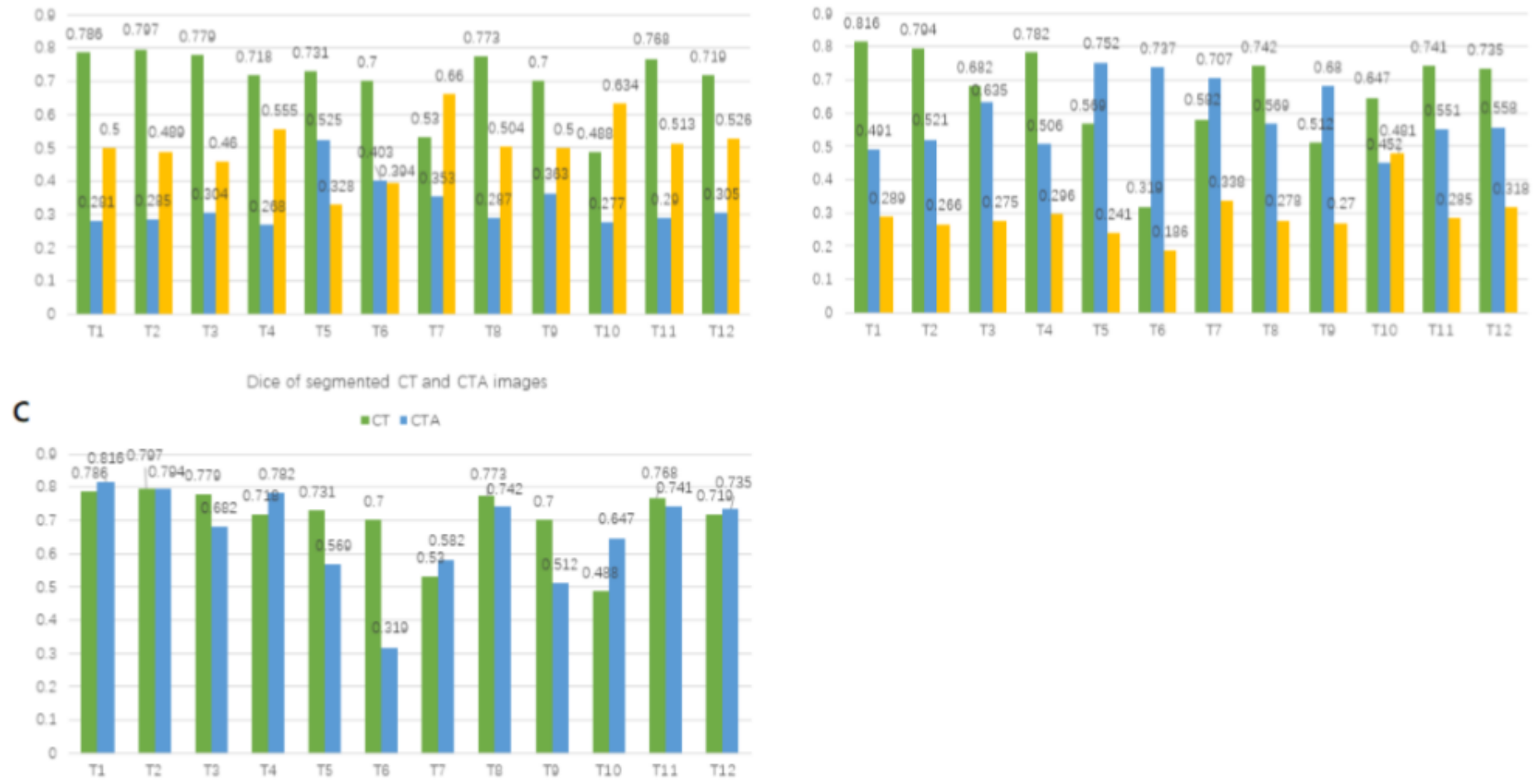

Figure 4

Dice coefficients and OR, UR results by each team. a-c.

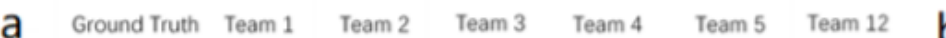

总 总

兽

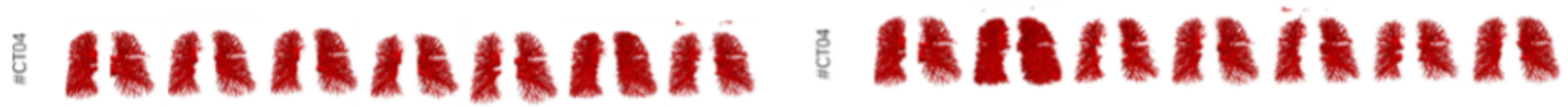

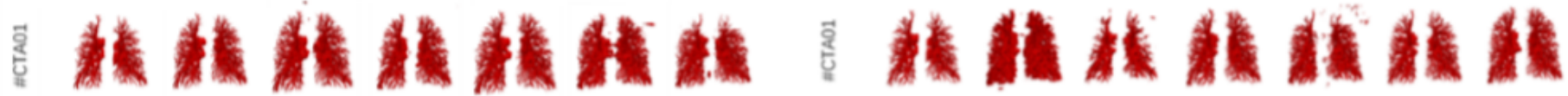

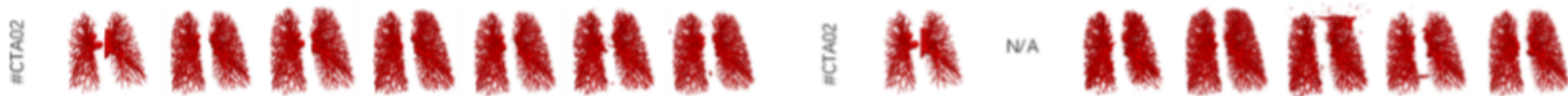
I

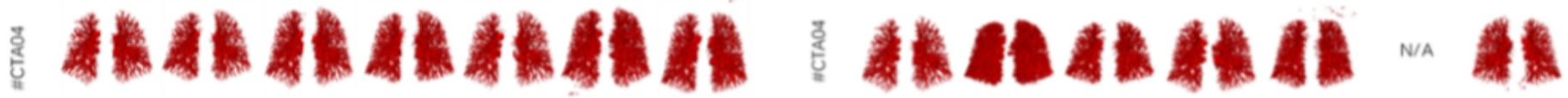


three-dimensional display for pulmonaty vascular segmentation results by each team. a-b. CT01-CT04 respectively represents four datasets for CTA images. The CTA02 result by team 5 is over segmented,which leads to insufficient memory to display normally, and the CTA04 result for team 10 is blank. 